\title{
Self-propulsion of a counter-rotating cylinder pair in a viscous fluid
}

\author{
Wim M. van Rees, ${ }^{1, a)}$ Guido Novati, ${ }^{1,2}$ and Petros Koumoutsakos ${ }^{1, b)}$ \\ ${ }^{1}$ Chair of Computational Science, ETH Zurich, Zurich, Switzerland \\ ${ }^{2}$ Department of Mechanical Engineering, Delft University of Technology, Delft, \\ The Netherlands
}

(Received 13 October 2014; accepted 25 April 2015; published online 12 June 2015)

\begin{abstract}
We study a self-propelling pair of steadily counter-rotating cylinders in simulations of a two-dimensional viscous fluid. We find two strikingly, opposite directions for the motion of the pair that is characterized by its width and rotational Reynolds number. At low Reynolds numbers and large widths, the cylinder pair moves similarly to an inviscid point vortex pair, while at higher Reynolds numbers and smaller widths, the pair moves in the opposite direction through a jet-like propulsion mechanism. Increasing further the Reynolds number, or decreasing the width, gives rise to non-polarised motion governed by the shedding direction and frequency of the boundary-layer vorticity. We discuss the fundamental physical mechanisms for these two types of motion and the transitions in the corresponding phase diagram. We discuss the fluid dynamics of each regime based on streamline plots, tracer particles, and the vorticity field. The counter rotating cylinder pair serves as a prototype for self-propelled bodies and suggests possible engineering devices composed of simple components and tunable by the rotation and width of the cylinder pair. ( 2015 AIP Publishing LLC. [http://dx.doi.org/10.1063/1.4922314]
\end{abstract}

\section{INTRODUCTION}

Steady locomotion of aquatic swimmers involves a body translating with respect to the surrounding fluid with a net zero hydrodynamic force. Natural swimmers at intermediate and high Reynolds numbers achieve this by alternating periods of thrust and drag through periodic body deformations such as undulations in fish or contractions in jellyfish. ${ }^{1-3}$ The physical mechanisms underlying these forms of propulsion are widely studied and mimicked for relevant engineering applications. ${ }^{4-6}$ Alternative forms of aquatic locomotion have been presented for a flapping body moving laterally due to vortical instabilities, ${ }^{7}$ and for a passive body in a flow due to fluid mediated interactions of a nearby actively controlled object. ${ }^{8}$

Here, we consider a simplified concept for locomotion formed by two steadily counter-rotating cylinders that are linked together by their centers of mass. This simple system is characterized by two parameters, the non-dimensional width and the rotational Reynolds number of the cylinders. The propulsion mechanism relies purely on viscous effects, since in potential flow, d'Alembert's paradox states that the force on the body is zero at all times.

The flow past a non-moving counter-rotating cylinder pair in a viscous fluid has been first investigated almost a century ago. ${ }^{9}$ Since then, several authors have presented extensive analytical studies for the behaviour of the flow at low Reynolds numbers. ${ }^{10,11}$ Numerical results for the flow past a fixed counter-rotating cylinder pair at non-zero Reynolds numbers were presented by Chan et al. ${ }^{12}$ They found that for certain combinations of inflow and rotational velocities, the vortical wake was

\footnotetext{
a) Present address: School of Engineering and Applied Sciences, Harvard University, USA.

b) Electronic mail: petros@ethz.ch
} 
suppressed resulting in zero or negative drag values. In the context of self-propulsion, steady-state solutions were found numerically for two counter-rotating cylinders ${ }^{13,14}$ and an axisymmetric torus. ${ }^{15}$ These numerical studies confirm the existence of two flow regimes. When the inner surfaces of the cylinders spin against the inflow direction, an elliptical region of circulating fluid around the entire pair can be established, whereas in the opposite rotational direction, closed streamlines around each of the cylinders are found. However, the unsteady locomotory behavior of the cylinder pair, as well as a systematic investigation of the cylinders' behavior in the space of governing parameters, has not been reported yet.

Here, we study the self-propelling cylinder pairs through unsteady simulations using a 2D multiresolution remeshed vortex method. This enables the study of transient behaviour and the emergence of instabilities in the system. Furthermore, we explore a wide phase space for the rotation and width of the pair. Using streamlines and passive tracer particles, we discuss the governing flow physics and report on the high Reynolds number instabilities.

In Sec. II, we describe the computational setup, followed by an overview of the results (Sec. III), a discussion of our findings from a hydrodynamic point of view (Sec. IV), and the conclusions in Sec. V.

\section{SETUP AND METHODS}

In this section, we first explain the problem setup and then introduce the computational method used for the flow simulations.

\section{A. Problem setup}

We consider two identical counter-rotating cylinders that are virtually connected by their respective centers of mass so as to move as a single rigid body, as sketched in Figure 1. The geometry is defined by the distance between the centers of mass of the cylinders $(W)$ and the diameter of the cylinders $(D)$. The cylinders rotate with equal but opposite angular velocity $\Omega$, with a corresponding circulation of strength $|\Gamma|=\pi|\Omega| D^{2} / 2$. This body is immersed in a fluid with viscosity $v$ and density $\rho_{f}$, which is equal to the body density $\rho_{s}$ so that gravitational effects can be ignored. Each setup can be uniquely characterized by the non-dimensional width $W^{*}=W / D$ and the Reynolds number $R e_{\Gamma}=|\Gamma| / v$, where $v$ is the kinematic viscosity of the fluid. The alternative Reynolds number definition $\operatorname{Re}_{\Omega}=|\Omega| D^{2} / v=\pi R e_{\Gamma} / 2$ can be easily obtained from $R e_{\Gamma}$. Throughout this work, we set a positive (counter-clockwise) angular velocity for the left cylinder. Time $t$ is non-dimensionalized using the period of revolution of the cylinders, $T=t|\Omega| /(2 \pi)=t|\Gamma| /\left(\pi^{2} D^{2}\right)$, and the velocity $u$ is non-dimensionalized using the surface velocity so that $U=2 u /(|\Omega| D)=u \pi D /|\Gamma|$.
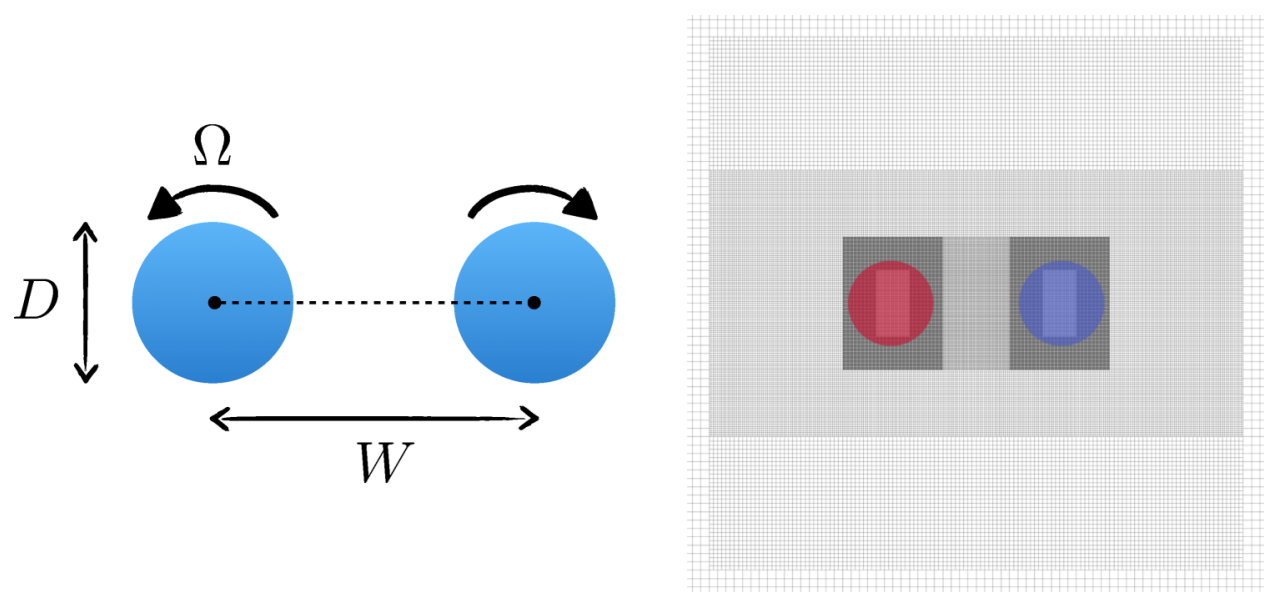

FIG. 1. Sketch of the problem setup (left) and a close-up of the initial wavelet-adapted grid with the two cylinders colored in red and blue, respectively (right). The problem is governed by two non-dimensional numbers, the Reynolds number $\operatorname{Re}_{\Gamma}=\Gamma / v=\pi|\Omega| D^{2} /(2 v)$, and the non-dimensional width $W^{*}=W / D$. 


\section{B. Computational method}

All simulations are performed with a 2D multiresolution remeshed vortex method using penalization and projection methods to account for the fluid-solid interaction. ${ }^{16}$ The method solves the vorticity equation on a wavelet-adapted computational grid, using a multipole-based solver to reconstruct the velocity from the vorticity field. ${ }^{17}$ Particles are used for the advection part of the equation; diffusion is handled with a fourth-order finite difference scheme and an explicit Runge-Kutta 2 time stepping scheme. The solver uses local time stepping whenever the diffusion time step restriction dominates. The method and code have been extensively used and validated for similar problems. ${ }^{8,16-19}$ Throughout this work, we set the effective resolution of our unit square domain to $E R=32768^{2}$, and the cylinder radius equal to $R=0.0025$, so that there are over 80 grid points across the radius at the maximum level of grid refinement. The refinement and compression thresholds are $r_{\text {tol }}=10^{-3}$, $c_{\mathrm{tol}}=10^{-4}$, and the cylinder is represented on the computational grid through a discrete Heaviside function. ${ }^{20}$ The time step criterion is LCFL $=0.05$ for all results presented here, and we ramp up the circulation strength of the cylinders with a quarter period of a sine function during their first rotation.

\section{RESULTS}

First, we show the result of varying $R e_{\Gamma}$ while keeping $W^{*}=2$, then we vary $W^{*}$ while fixing $R e_{\Gamma}=100$. Subsequently, we show the characteristic behavior of the cylinders as a function of both $\operatorname{Re}_{\Gamma}$ and $W^{*}$.

\section{A. Varying $R e_{\Gamma}$ with $W^{*}=2$}

The translational body velocity for different Reynolds numbers at a fixed non-dimensional width of $W^{*}=2$ is shown in Figure 2. For Reynolds numbers up to $R e_{\Gamma}=85$, the cylinders move upwards, reaching higher steady-state velocities as the Reynolds number increases. At $R e_{\Gamma}=90$, the cylinders accelerate upwards for the first five revolutions but then the acceleration gradually changes direction and remains negative, so that the cylinders eventually move in downward direction and reach a negative steady-state velocity. For higher Reynolds numbers, the cylinders are consistently moving downwards, reaching higher steady-state speeds and larger initial acceleration magnitudes as the Reynolds number increases. The right plot in Figure 2 shows the vertical velocity of the cylinders for Reynolds numbers above $R e_{\Gamma}=100$. We can observe that the downwards velocity magnitude increases until $R e_{\Gamma}=500$ but oscillations in the velocity curve appear. At $R e_{\Gamma}=625$ and $R e_{\Gamma}=750$, the cylinders oscillate strongly upwards and downwards without reaching steady-state within the first 125 revolutions.
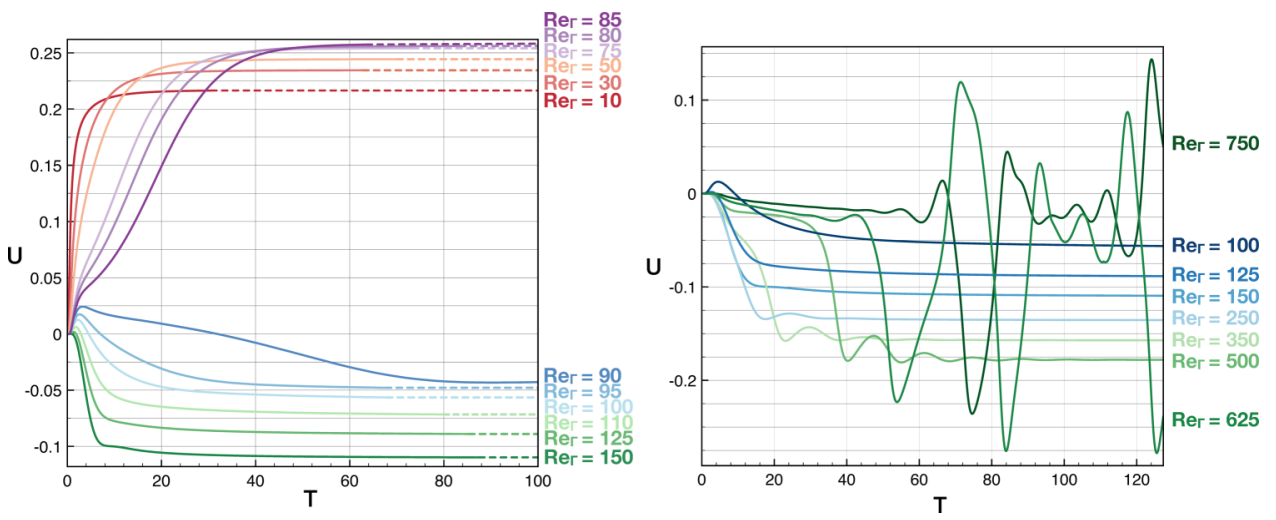

FIG. 2. Velocity of the counter-rotating cylinder pair at $W^{*}=2$ at a series of Reynolds numbers up to $\operatorname{Re}_{\Gamma}=150$ (left) and up to $R e_{\Gamma}=750$ (right). For the left plot, simulations are stopped whenever the velocity does not change more than $0.03 \%$ in the last time unit, and extended with a dashed line to indicate the steady-state value. 


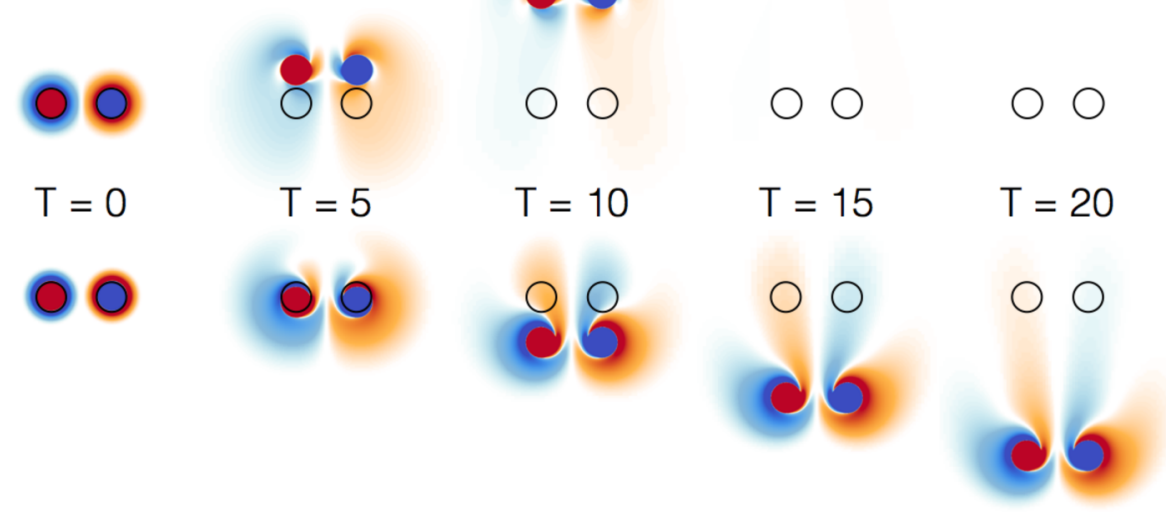

FIG. 3. Vorticity field of the counter-rotating cylinder pair at $W^{*}=2$ at $R e_{\Gamma}=50$ (top) and $R e_{\Gamma}=150$ (bottom, separate simulation) at different times. The initial location of the cylinder pair is drawn with solid black circles. (Multimedia view) [URL: http://dx.doi.org/10.1063/1.4922314.1]

Representative vorticity fields corresponding to the upwards $\left(R e_{\Gamma}=50\right)$ and downwards $\left(R e_{\Gamma}=\right.$ 150) regime are shown in Figure 3. At steady-state the vorticity field at $R e_{\Gamma}=50$ is mostly contained around the cylinders, and the strongest vorticity values are those corresponding to the cylinders' rotations. In contrast, at $R e_{\Gamma}=150$, a wake is clearly visible corresponding to a strong flow in between the cylinders, and the highest vorticity magnitudes are just outside of the cylinder surfaces on the inside of the pair.

\section{B. Varying $W^{*}$ with $R e_{\Gamma}=100$}

In Figure 4, we present the vertical velocity curves for a fixed Reynolds number of $\operatorname{Re}_{\Gamma}=100$ while varying the non-dimensional width $W^{*}$. In this case, the cylinders move upwards for $W^{*} \leq 1.75$ and downwards for $W^{*} \geq 2$. Increasing the value of $W^{*}$ even further results in unstable behavior as the cylinders alternate between upwards and downwards motions.


FIG. 4. Velocity of the counter-rotating cylinder pair at $\operatorname{Re}_{\Gamma}=100$ for a series of non-dimensional widths $W^{*}$. Simulations are stopped whenever the velocity does not change more than $0.03 \%$ in the last time unit, and extended with a dashed line to indicate the steady-state value. 


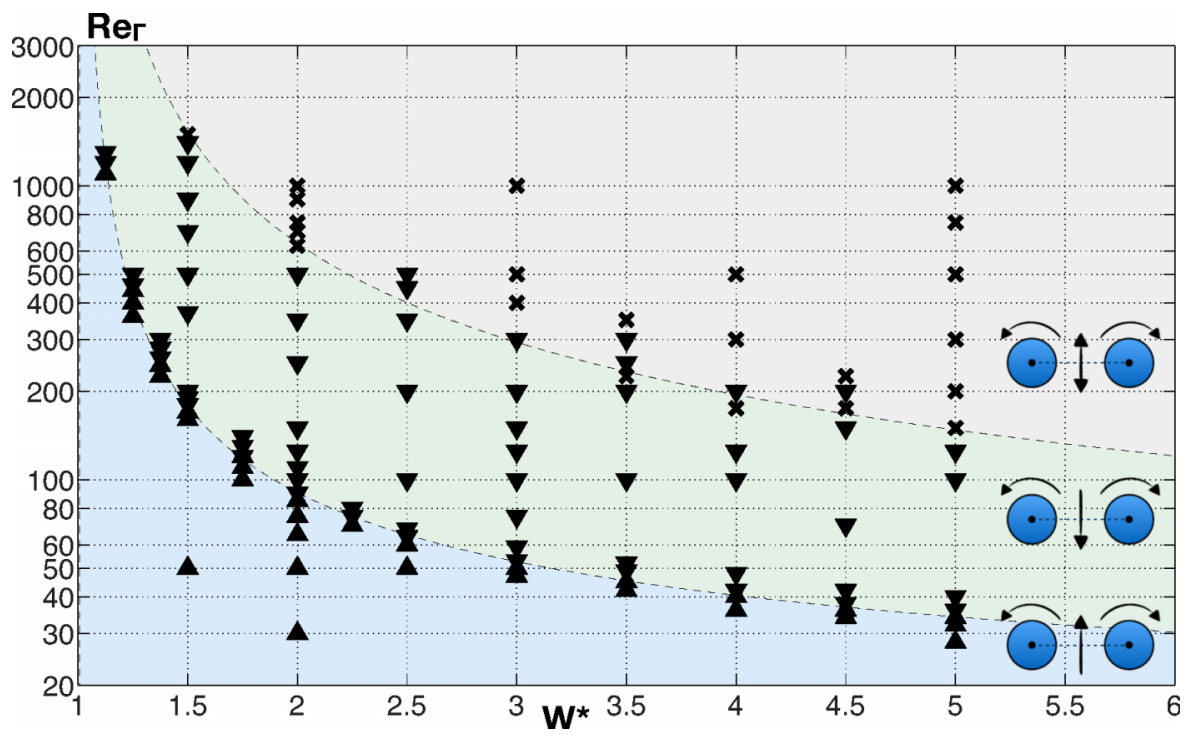

FIG. 5. Transition between upward, vortex pair-like motion (upwards pointing triangles), and downward, jet-like motion (downwards pointing triangles) as a function of $\operatorname{Re}_{\Gamma}=\Gamma / v$ and $W^{*}=W / D$. The crosses denote unstable motion.

\section{Phase-space results}

To quantify the transition between upwards and downwards motion, as well as between stable and unstable motion, we performed a series of computations varying both $R e_{\Gamma}$ and $W^{*}$. Each computation is run until steady-state and the trend is noted. The points in the $R e_{\Gamma}-W^{*}$ space are chosen manually, both to accurately capture the change in motion direction and to cover the parameter space. A flow case is determined unstable if oscillations in the velocity evolution are observed which do not dampen out during the first $T=100$ cylinder revolutions.

The results, presented in Figure 5, show that the transition between upwards and downwards motion occurs at lower $R e_{\Gamma}$ values when the non-dimensional width is increased. Similarly, for small values of $W^{*}$, the transition happens at very large Reynolds numbers. The extreme cases, towards very small or very large widths, could not be simulated due to computational restrictions; however, for any width within $1.25 \leq W^{*} \leq 10$, we found the upward/downward transition occurring at a finite Reynolds number.

We provide an empirical description of the boundary between upwards and downwards motion using a curve-fitting based on an exponential relationship. The transition points are defined for each $W^{*}$ as the average between the largest $R e_{\Gamma}$ resulting in upwards motion and the smallest $\operatorname{Re}_{\Gamma}$ resulting in downwards motion. We fit the parameters of the function

$$
\operatorname{Re}_{\Gamma}=a \exp \left[\left(\frac{b}{\left(W^{*}-1\right)}\right)^{c}\right],
$$

resulting in the parameters $a=6.7, b=17.5$, and $c=0.33$.

The instability can also be characterized by an exponential function of $W^{*}$; in this case, we find $a=13, b=56$, and $c=0.33$. Here, the fit deviates further from the data point, as the onset of an instability is harder to identify. Nevertheless again within the range of $1.25 \leq W^{*} \leq 10$, we could always find a high enough Reynolds number for which the velocity of the cylinders was unstable.

\section{DISCUSSION}

We discuss the steady locomotion of the cylinder pair in terms of the flow fields presented in Sec. III. We also discuss the transition between upwards and downwards motion, and the transition from downwards/jet-like motion to unstable solutions. 


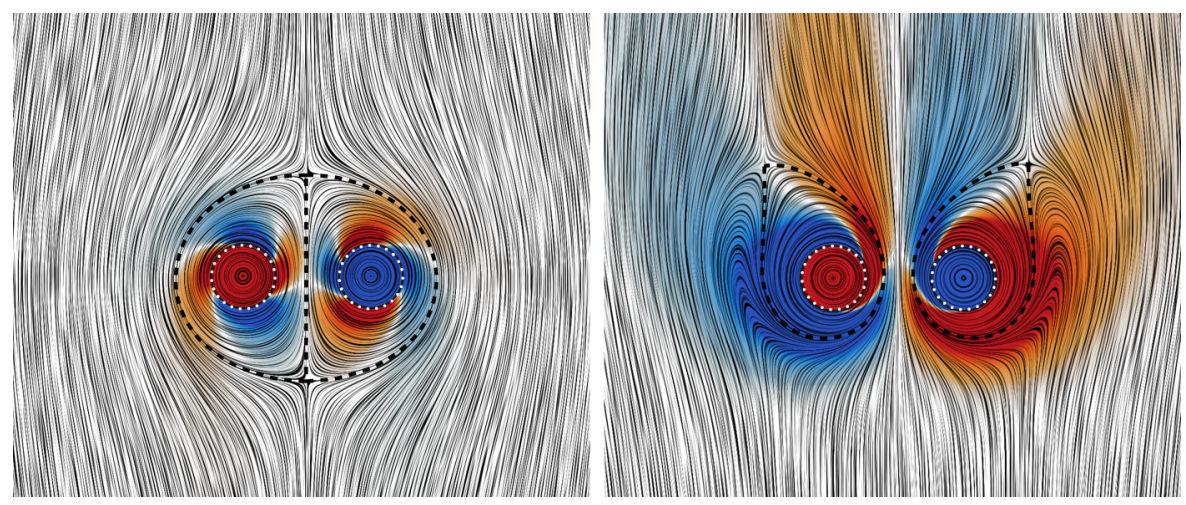

FIG. 6. Line-integral convolution field ${ }^{21}$ illustrating the velocity field around the cylinders at Reynolds number 50 (left) and 150 (right), computed in the moving frame of reference of the cylinders. The cylinders are marked by dotted white lines, closed streamlines around the cylinders by dashed black lines and the colors refer to the vorticity field.

\section{A. Flow visualization}

Figure 6 visualizes the velocity field of the cylinders at steady-state using a Line Integral Convolution (LIC) ${ }^{21}$ which can be interpreted as a space-filling streamline plot. We show the LIC field both for $R e_{\Gamma}=50$ and $R e_{\Gamma}=150$ in the frame of reference of the body center of mass. The lower Reynolds number solution shows a coherent elliptical region of circulating fluid surrounding the cylinder pair, traveling upwards with the cylinders, similar to the streamlines around a vortex pair moving at steady state. This is similar to the flow obtained past a stationary rotating cylinder pair with suppressed wake. ${ }^{12}$ In contrast, at the higher Reynolds number, there is a closed region of fluid around each cylinder. The incoming flow is distributed around and in between the cylinders, reminiscent of a jet-like flow where the fluid is ejected through the opening between the cylinders. These results are consistent with Refs. 13 and 14.

We also examine the two flow regimes discussed above by seeding the flow with tracer particles that are passively advected with the local velocity field. The evolution of the particles is shown in Figure 7. The particles confirm the observations made based on the streamlines: for the lower Reynolds number, corresponding to upwards motion, the surrounding flow does not penetrate the

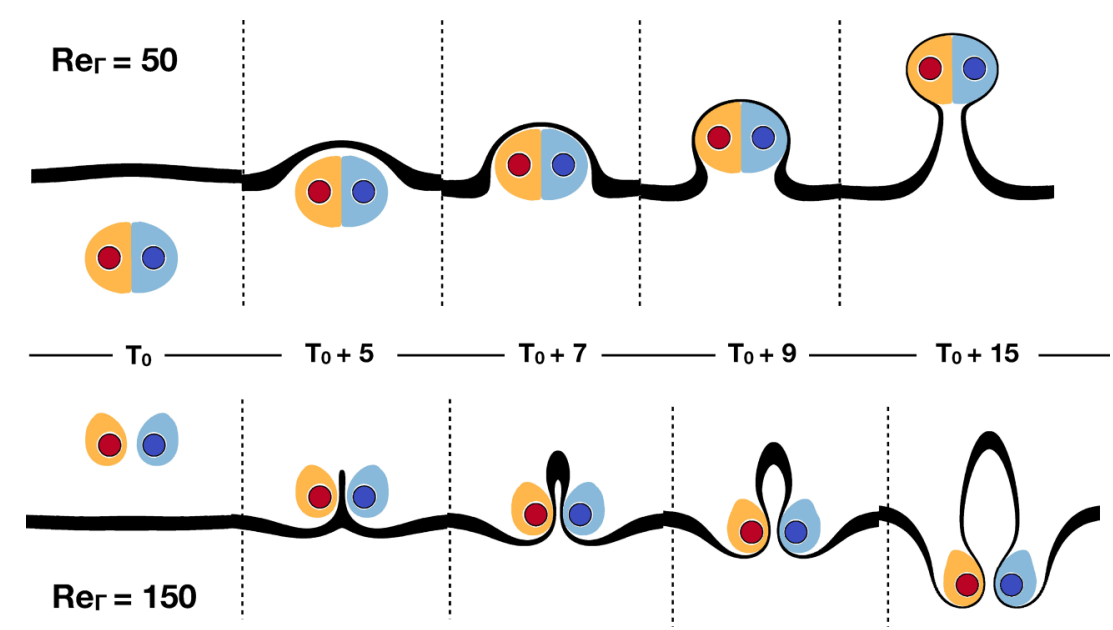

FIG. 7. Passive tracer particles are advected with the velocity field around the cylinders at $R e_{\Gamma}=50$ (top) and $R e_{\Gamma}=150$ (bottom). The cylinders move at steady-state and are depicted by the dark red and dark blue circles (left and right, respectively). We seed passive tracer particles around the counter-clockwise rotating cylinder (in light red/left), around the clockwise rotating cylinder (in light blue/right), and in a strip upstream of the cylinder (in black). (Multimedia view) [URL: http://dx.doi.org/10.1063/1.4922314.2] 


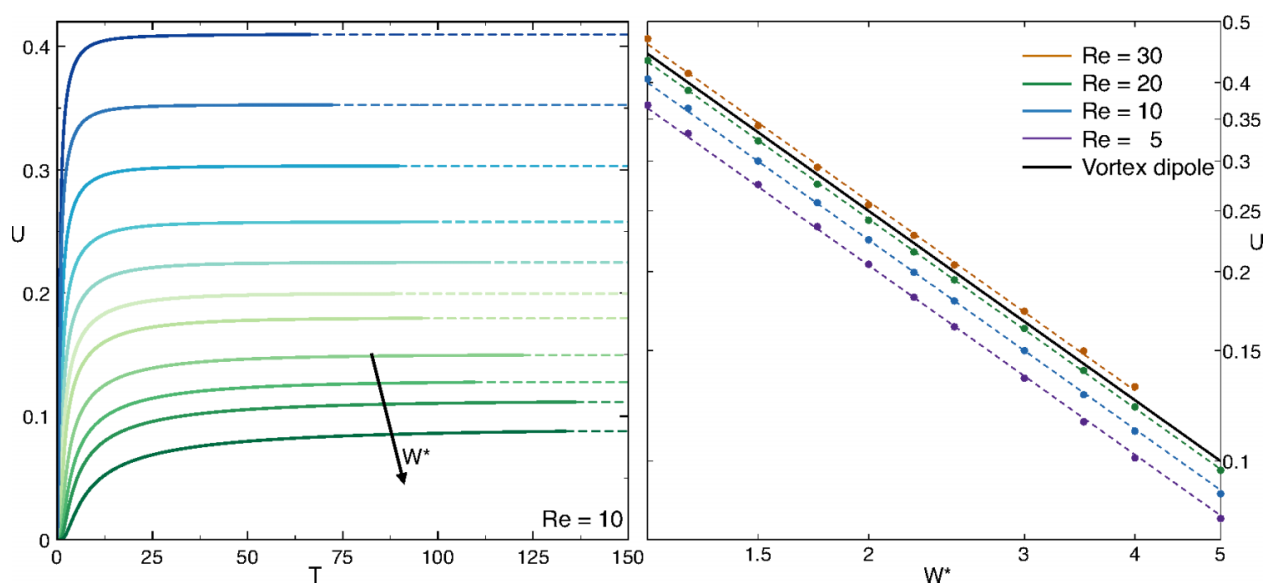

FIG. 8. On the left, velocity of the cylinder pair at $\operatorname{Re}_{\Gamma}=10$ for a series of $W^{*}$. From blue to green: $W^{*}=$ $[1.125,1.25,1.5,1.75,2.0,2.25,2.5,3.0,3.5,4.0,5.0]$. On the right, a logarithmic plot of the steady-state velocity against the distance $W^{*}$ between the cylinders for various $\operatorname{Re}_{\Gamma}$ compared to the velocity for a ideal vortex pair (black). The associated video shows vorticity field in selected cases. (Multimedia view) [URL: http://dx.doi.org/10.1063/1.4922314.3]

closed region of fluid surrounding the cylinder pair, instead it is being deflected around it. At higher Reynolds number, the particles are pushed between the two cylinders in a jet-type flow.

\section{B. Low Reynolds number regime}

To compare the lower Reynolds number flow regime to the idealized case of a point vortex pair, we consider the velocity of the vortex pair as

$$
U=\frac{u}{U_{\theta}}=\frac{\Gamma /(2 \pi W)}{U_{\theta}}=\frac{1}{2 W^{*}},
$$

where we expressed the circulation $\Gamma$ through the corresponding surface velocity for a cylinder of diameter $D$. In Figure 8 (left), we plot the velocity of the cylinder pair at $R e_{\Gamma}=10$ as a function of $W^{*}$, and observe that the steady-state velocity has an inversely linear relation to $W^{*}$. The right plot of Figure 8 indeed confirms that the steady-state velocity at various Reynolds numbers below the transition is inversely proportional to the non-dimensional width, similar to a vortex pair. Linear fitting the data points gives prefactors of $(0.41,0.45,0.485,0.518)$ for Reynolds numbers $(5,10,20$, 30 ), very close to the inviscid value of 0.5 .

\section{High Reynolds number regime}

For the higher Reynolds number regime, the propulsion of the cylinders relies on the acceleration of the fluid moving in between them. The streamline pattern of this regime resembles that of an actuator disc (Figure 6), which is typically used to model the thrust of traditional propellers. In our setup, the gap between the cylinders can correspondingly be considered an actuator line. The flow acceleration through the gap is driven by the viscous shear at the cylinders' surfaces, reminiscent of a transient symmetric Couette flow. Currently, the spatial and temporal scales over which the flow is accelerated through shear are still unknown. Nevertheless, with the addition of an accurate expression for the drag coefficient, this line of thought could provide a starting point for modeling the cylinders' behavior in this regime.

\section{Transition between the regimes}

As discussed in the previous two paragraphs, the low Reynolds number regime reminds of a point vortex pair, whereas the high Reynolds number regime resembles a shear-driven actuator disc. 
Similar to the streamline pattern of these two fundamental flows, in the low Reynolds number regime, the external flow moves around the cylinder pair, whereas in the high Reynolds number regime, the external flow is accelerated in between the two cylinders (Figure 6). To explain the transition between these states, we consider the thickness of the boundary-layer on each cylinder with respect to the gap between the cylinders. In the low Reynolds number regime, this ratio is large, so that the rotational flow induced by the cylinders' motion is sustained in the entire width of the gap. Therefore, the closed streamline pattern of Figure 6 can be realized, so that the vortex pair-like motion is achieved. As the Reynolds number increases, the boundary-layer thickness around each cylinder, in relation to the gap between the cylinders, decreases. Consequently, some of the fluid in between the cylinders, instead of being carried around each cylinder, is expelled into the wake and forms a jet.

This explanation holds both for an increase in rotational Reynolds number at constant width, and for an increase in width at constant rotational Reynolds number. In both cases, the thickness of the boundary layers decreases with respect to the gap between the two cylinders, and a transition between the two regimes occurs.

\section{E. Flow instability at high Reynolds numbers}

The transition to unstable motion is shown in more detail in Figure 9, for $W^{*}=3$ and $W^{*}=5$, at a variety of Reynolds numbers. For $W^{*}=3$, starting at $R e_{\Gamma}=125$, the cylinders move downwards in stable manner. As the Reynolds number increases, the cylinders' initial acceleration decreases causing an accumulation of vorticity near the surface. This shear layer develops an instability that grows as it is convected along the surface. Eventually, the instability causes the boundary-layer vorticity to detach and as it is shed into the wake, the cylinders accelerate in opposite direction. With increasing Reynolds number, the momentum of the fluid shed into the wake increases, so that the cylinders are gradually accelerated faster by this process. This is visible in the $W^{*}=3$ plot as growing oscillations amplitudes between $T=25$ and $T=50$ for $R e_{\Gamma}=150$ up to $R e_{\Gamma}=300$. For all these cases, however, the cylinder manages to restore to a steady velocity in downward direction. At $R e_{\Gamma}=500$, this capacity is lost and the cylinders behave unpredictably. For such high Reynolds numbers, the growth rate of the instability is too large for the cylinders to settle at a steady-state. Since the trigger for the initial perturbation in our setup is caused by non-reproducible floating-point arithmetic, repeated simulations in this unstable regime can give drastically different outcomes.

The cylinder pair with $W^{*}=5$ has a qualitatively similar behavior; in this case, the cylinders become unstable at $R e_{\Gamma}=200$. Surprisingly, though, at $R e_{\Gamma}=300$, the pair has recovered a seemingly stable solution where the motion direction is upwards, and vorticity is shed continuously into the wake. Then at $R e_{\Gamma}=500$, the cylinders initially are unstable until the velocity finally keeps hovering around a negative value. The LIC field of the latter two cases is shown in Figure 10. The velocity field
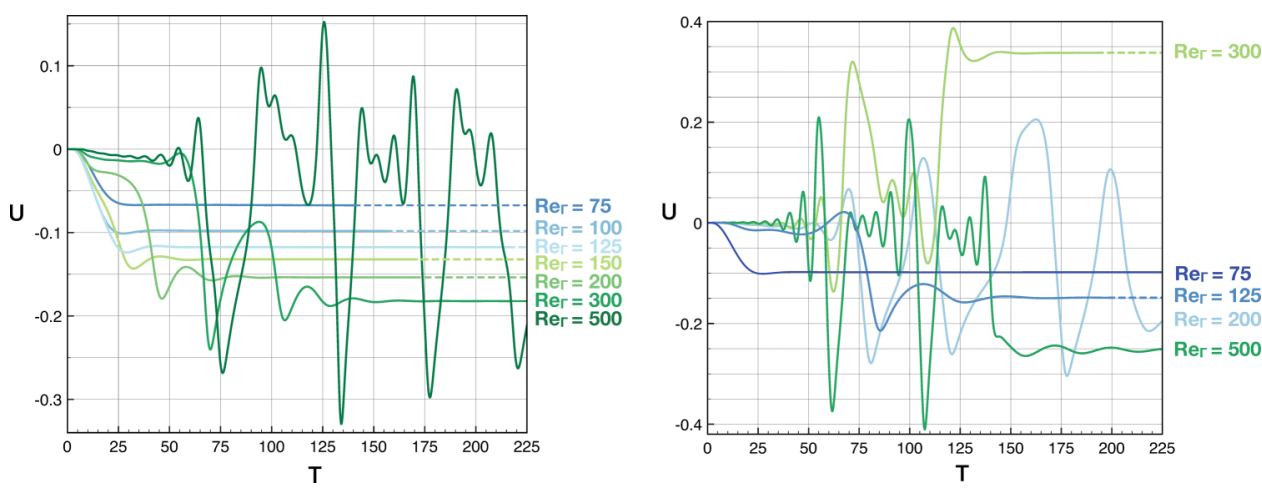

FIG. 9. Vertical velocity of the cylinders with $W^{*}=3$ (left) and $W^{*}=5$ for Reynolds numbers around the instability. The associated video shows vorticity field for selected cases at $W^{*}=3$. (Multimedia view) [URL: http://dx.doi.org/ 10.1063/1.4922314.4] 


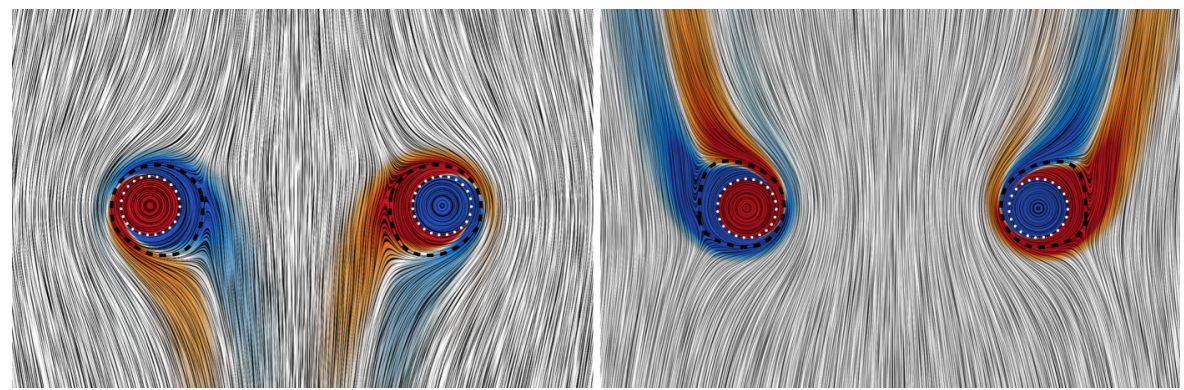

FIG. 10. Line-integral convolution field ${ }^{21}$ of the velocity around the cylinders with $W^{*}=5$ at $R e_{\Gamma}=300$ (left) and $R e_{\Gamma}=500$ (right), computed in the moving frame of reference of the cylinders. The cylinders are marked by dotted white lines, closed streamlines around the cylinders by dashed black lines, and the colors refer to the vorticity field. (Multimedia view) [URL: http://dx.doi.org/10.1063/1.4922314.5]

at $R e_{\Gamma}=500$ is comparable with that of the low Reynolds number case in Figure 6, where both cylinders move downwards. However at $R e_{\Gamma}=300$, the flow is very different from the previous upward solution. The closed elliptical region of flow surrounding the cylinder pair is absent, instead the flow travels in between the cylinders.

Interestingly, a quasi-periodic case was found for the evolution of $W^{*}=3$ and $R e_{\Gamma}=1000$, as shown in Figure 11. These cylinders show a quasi-periodic vortex shedding that propels them forward incrementally. After a vortex pair has been shed, the boundary-layer instability builds up afresh until it finally sheds. With this pattern, the cylinders manage to move around 40 diameters during 300 revolutions.

\section{F. Transient behavior}

To investigate the stability of the steady-state solutions of each regime, we consider the case of a change in circulation after the cylinder pair has reached steady state in either regimes. Specifically,
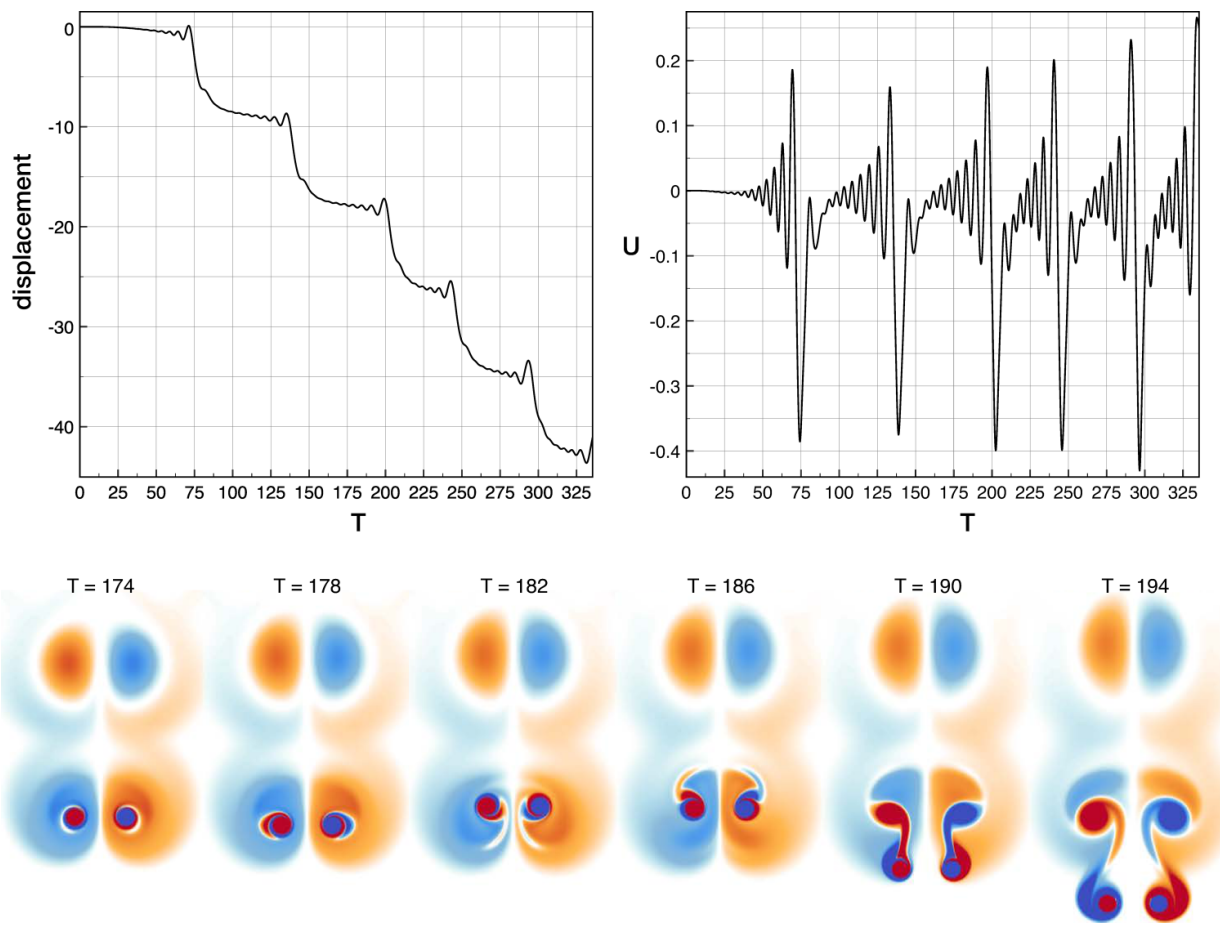

FIG. 11. Displacement (top left, non-dimensionalized with the diameter $D$ ), velocity (top right) and vorticity field (bottom) for the cylinders with $W^{*}=3$ and $R e_{\Gamma}=1000$. (Multimedia view) [URL: http://dx.doi.org/10.1063/1.4922314.6] 

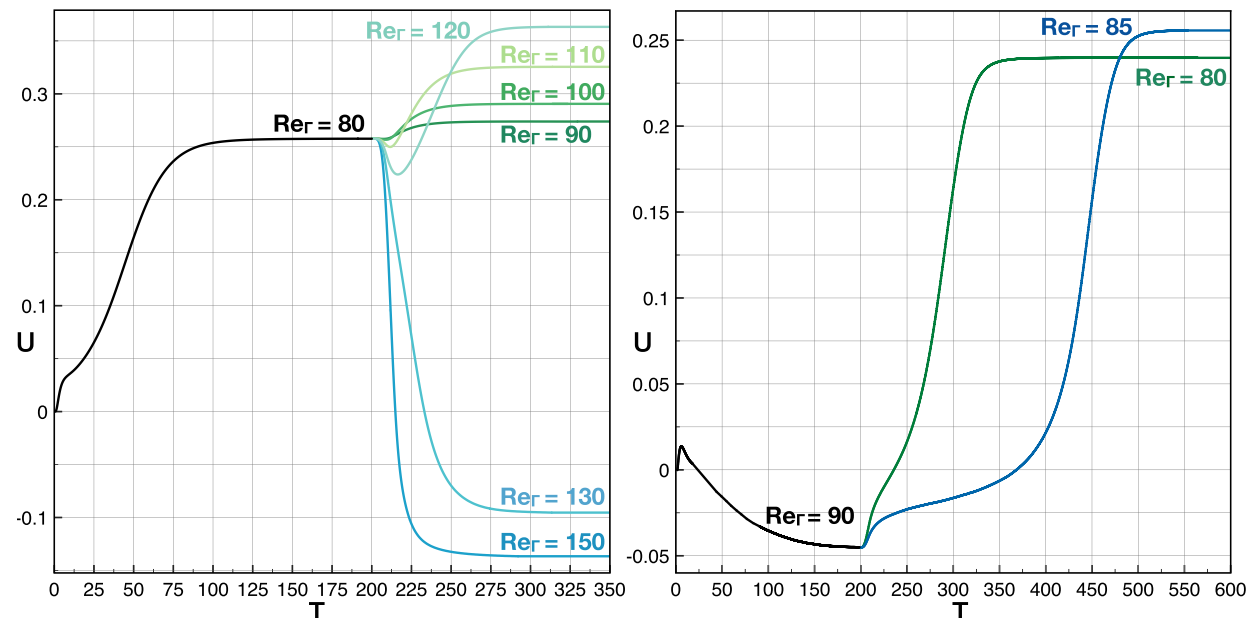

FIG. 12. Cylinder pair behavior in response to an increase in circulation starting from $\operatorname{Re}_{\Gamma}=80$ (left), and to a decrease in circulation starting from $R e_{\Gamma}=90$ (right). The width is $W^{*}=2$. The associated video shows vorticity field in selected cases. (Multimedia view) [URL: http://dx.doi.org/10.1063/1.4922314.7]

we change the circulation magnitude of both cylinders according to

$$
\Gamma(T)= \begin{cases}\Gamma_{0}, & T \leq T_{0}, \\ \Gamma_{0}\left(1+\frac{1}{2}\left(\frac{\Gamma_{1}}{\Gamma_{0}}-1\right)\left[1-\cos \left(\pi \frac{T-T_{0}}{T_{1}-T_{0}}\right)\right]\right), & T_{0}<T<T_{1}, \\ \Gamma_{1}, & T \geq T_{1},\end{cases}
$$

where the non-dimensionalization is based on the state values at $T \leq T_{0}$. This equation secures a smooth transition from circulation $\Gamma_{0}$ to $\Gamma_{1}$ between times $T_{0}=200$ and $T_{1}=210$ with a half-period cosine function.

In Figure 12, we show what happens when the cylinder pair is subjected to an increase in circulation when starting from a vortex pair-like regime (left), and to a decrease in circulation when starting from a jet-like regime (right). In the former case, we see that the vortex pair-like regime can be extended to much higher Reynolds numbers when starting from a steady-state solution. At $\operatorname{Re}_{\Gamma}=120$, we observe that during the transition, the cylinder pair initially slows down, yet after the new circulation value is reached, it speeds up again. The steady-state at $R e_{\Gamma}=120$ corresponds to a $40 \%$ larger velocity than the steady-state at $R e_{\Gamma}=80$.

\section{CONCLUSIONS}

In this work, we discussed a simple mechanism for self-propulsion in a viscous fluid consisting of a steadily counter-rotating cylinder pair. For low rotation rates, the cylinders behave as an ideal vortex pair, moving in the direction of the internal surface velocity. The flow is characterized by an elliptical region of fluid surrounding the cylinders enclosed by a bounding streamline, around which the external fluid is deflected. We demonstrated that in this regime, the velocity of the cylinders is inversely proportional to the width, as for the ideal vortex pair. Increasing the rotation rate shows a transition to a new state where the cylinders move in the opposite direction. In this regime, the external flow is accelerated in between the cylinders and expelled like a jet. The Reynolds number of transition between these two states depends on the width between the cylinders with an inverted exponential relationship, so that larger widths require smaller rotation rates to move from the vortex-pair-type to the jet-type flow.

Increasing the rotation rate even further shows an unstable regime as the instabilities growing in the boundary layer of each cylinder are shed into the wake. For small rotation rate increments, the cylinders can recover from the first shedding event to settle in a steadily moving jet-like state. 
At higher rotation rates, their motion is dominated by discrete vortex shedding events, each of which may result in upwards or downwards motion depending on the direction in which the vorticity is shed. This generally results in unsteady, irregular behavior but we demonstrate that some isolated stable or quasi-periodic solutions exist within the unstable regime, which are sustained for longer periods of time.

We believe this simplified form of locomotion can serve as inspiration for the development of engineering devices in fields such as robotics and fluid mixing. Changing the rotation of one of the cylinders can enable the system to rotate, a useful property for robotic devices. Future work will investigate the energy efficiency and performance when the cylinders' rotation has an oscillatory character. Furthermore, we will consider the extension of this system to three dimensions. On the latter subject, one can imagine a variety of geometric configurations, ranging from simply a pair of rotating spheres to planar triangular configurations with rotating spheres on the vertices, or rotating cylinders on the edges of morphing structures, a subject of ongoing investigations in our lab.

\section{ACKNOWLEDGMENTS}

W. M. V. R. wishes to thank the Swiss National Science Foundation (SNF) for financial support. G. N. wishes to acknowledge the IDEA League for the scholarship that financially supported his stay in Zurich. P.K. acknowledges support by the Advanced Grant of the European Research Council (Grant No: 341117). We thank the reviewers for their helpful comments and suggestions.

${ }^{1}$ S. Childress, Mechanics of Swimming and Flying (Cambridge University Press, 1981).

${ }^{2}$ M. S. Triantafyllou, G. S. Triantafyllou, and D. K. P. Yue, "Hydrodynamics of fishlike swimming," Annu. Rev. Fluid Mech. 32, 33-53 (2000).

${ }^{3}$ T. Y. Wu, "Fish swimming and bird/insect flight," Annu. Rev. Fluid Mech. 43, 25-58 (2011).

${ }^{4}$ M. S. Triantafyllou and G. S. Triantafyllou, “An efficient swimming machine," Sci. Am. 272, 64-70 (1995).

${ }^{5}$ G. V. Lauder, E. J. Anderson, J. Tangorra, and P. Madden, "Fish biorobotics: Kinematics and hydrodynamics of selfpropulsion,” J. Exp. Biol. 210, 2767-2780 (2007).

${ }^{6}$ R. W. Whittlesey, S. Liska, and J. O. Dabiri, "Fish schooling as a basis for vertical axis wind turbine farm design," Bioinspiration Biomimetics 5, 035005 (2010).

${ }^{7}$ S. Alben and M. Shelley, "Coherent locomotion as an attracting state for a free flapping body," Proc. Natl. Acad. Sci. U. S. A. 102, 11163-11166 (2005).

${ }^{8}$ M. Gazzola, C. Mimeau, A. Tchieu, and P. Koumoutsakos, "Flow mediated interactions between two cylinders at finite Re numbers," Phys. Fluids 24, 043103 (2012).

${ }^{9}$ G. Jeffery, "The rotation of two circular cylinders in a viscous fluid," Proc. R. Soc. London, Ser. A 101, 169-174 (1922).

${ }^{10}$ E. Watson, "The rotation of two circular cylinders in a viscous fluid," Mathematika 42, 105-126 (1995).

${ }^{11}$ Y. Ueda, A. Sellier, T. Kida, and M. Nakanishi, "On the low-reynolds-number flow about two rotating circular cylinders," J. Fluid Mech. 495, 255-281 (2003)

${ }^{12}$ A. Chan, P. Dewey, A. Jameson, C. Liang, and A. Smits, "Vortex suppression and drag reduction in the wake of counterrotating cylinders," J. Fluid Mech. 679, 343-382 (2011).

${ }^{13}$ S. Sungnul and N. Moshkin, "Effect of rotation rates and gap spacing on the structure of low Reynolds number flow over two rotating circular cylinders," in Computational Fluid Dynamics 2008, edited by H. Choi, H. Choi, and J. Yoo (Springer Berlin Heidelberg, 2009), pp. 771-777.

${ }^{14}$ P. Suwannasri, "Numerical simulation of self-propelled motion of two rotating side-by-side circular cylinders," SoutheastAsian J. Sci. 2, 19-26 (2013).

${ }^{15}$ N. P. Moshkin and P. Suwannasri, "Two regimes of self-propelled motion of a torus rotating about its centerline in a viscous incompressible fluid at intermediate Reynolds numbers," Phys. Fluids 24, 053603 (2012).

${ }^{16}$ M. Gazzola, P. Chatelain, W. M. van Rees, and P. Koumoutsakos, "Simulations of single and multiple swimmers with non-divergence free deforming geometries," J. Comput. Phys. 230, 7093-7114 (2011).

${ }^{17}$ D. Rossinelli, B. Hejazialhosseini, W. M. van Rees, M. Gazzola, M. Bergdorf, and P. Koumoutsakos, "MRAG-I2D: Multi-resolution adapted grids for remeshed vortex methods on multicore architectures," J. Comput. Phys. 288, 1-18 (2015).

${ }^{18}$ M. Gazzola, W. M. van Rees, and P. Koumoutsakos, “C-start: Optimal start of larval fish,” J. Fluid Mech. 698, 5-18 (2012).

${ }^{19}$ W. M. van Rees, M. Gazzola, and P. Koumoutsakos, "Optimal shapes for intermediate Reynolds number anguilliform swimming," J. Fluid Mech. 722, R3 (2013).

${ }^{20}$ J. Towers, "Finite difference methods for approximating Heaviside functions," J. Comput. Phys. 228, 3478-3489 (2009).

${ }^{21}$ B. Cabral and L. Leedom, "Imaging vector fields using line integral convolution," in SIGGRAPH '93: Proceedings of the 20th Annual Conference on Computer Graphics and Interactive Techniques (Anaheim, CA), edited by S. Cunningham (Association for Computing Machinery, 1993), pp. 263-270. 